\title{
4D RECONSTRUCTION AND VISUALIZATION OF CULTURAL HERITAGE: ANALYZING OUR LEGACY THROUGH TIME
}

\author{
Pablo Rodríguez-Gonzálvez ${ }^{\mathrm{a}}$, Angel Luis Muñoz-Nieto ${ }^{\mathrm{a}}$, Susana del Pozo ${ }^{\mathrm{a}}$, Luis Javier Sanchez-Aparicio ${ }^{\mathrm{a}}$, \\ Diego Gonzalez-Aguilera ${ }^{\mathrm{a}}$, \\ Laura Micoli ${ }^{\mathrm{b}}$, Sara Gonizzi Barsanti ${ }^{\mathrm{b}}$, Gabriele Guidi ${ }^{\mathrm{b}}$, \\ Jon Mills ${ }^{\mathrm{c}}$, Karolina Fieber ${ }^{\mathrm{c}}$, Ian Haynes ${ }^{\mathrm{d}}$, \\ Beata Hejmanowska ${ }^{e}$
a Department of Cartographic and Land Engineering; High School of Ávila, University of Salamanca, Ávila, Spain - (pablorgsf, almuni, s.p.aguilera, luisj, daguilera)@usal.es
${ }^{\mathrm{b}}$ Dept. of Mechanical Engineering, Politecnico di Milano, Milan, Italy - (laura.micoli, sara.gonizzi, gabriele.guidi)@polimi.it
${ }^{\mathrm{c}}$ School of Civil Engineering and Geosciences, Newcastle University, Newcastle upon Tyne, UK - (jon.mills, Karolina.Fieber)@newcastle.ac.uk
${ }^{\mathrm{d}}$ School History, Classics and Archaeology, Newcastle University, Newcastle upon Tyne, UK - ian.haynes@newcastle.ac.uk
${ }^{\mathrm{e}}$ Stanislaw Staszic Scientific Association, Krakow, Poland - geo-staszic@gmail.com

\section{Commission II}

KEY WORDS: 4D Modelling, Cultural Heritage, Data Fusion, Monitoring, Visualization

\begin{abstract}
:
Temporal analyses and multi-temporal 3D reconstruction are fundamental for the preservation and maintenance of all forms of Cultural Heritage $(\mathrm{CH})$ and are the basis for decisions related to interventions and promotion. Introducing the fourth dimension of time into three-dimensional geometric modelling of real data allows the creation of a multi-temporal representation of a site. In this way, scholars from various disciplines (surveyors, geologists, archaeologists, architects, philologists, etc.) are provided with a new set of tools and working methods to support the study of the evolution of heritage sites, both to develop hypotheses about the past and to model likely future developments. The capacity to "see" the dynamic evolution of $\mathrm{CH}$ assets across different spatial scales (e.g. building, site, city or territory) compressed in diachronic model, affords the possibility to better understand the present status of $\mathrm{CH}$ according to its history. However, there are numerous challenges in order to carry out 4D modelling and the requisite multi-data source integration. It is necessary to identify the specifications, needs and requirements of the $\mathrm{CH}$ community to understand the required levels of 4D model information. In this way, it is possible to determine the optimum material and technologies to be utilised at different $\mathrm{CH}$ scales, as well as the data management and visualization requirements. This manuscript aims to provide a comprehensive approach for $\mathrm{CH}$ time-varying representations, analysis and visualization across different working scales and environments: rural landscape, urban landscape and architectural scales. Within this aim, the different available metric data sources are systemized and evaluated in terms of their suitability.
\end{abstract}

\section{INTRODUCTION}

Cultural Heritage $(\mathrm{CH})$ encompasses both tangible assets (e.g. monuments, archaeological remains, artefacts, etc.) and intangible ones (e.g. traditions, social practices, rituals, etc.). This paper focuses on the analysis of the tangible legacy. $\mathrm{CH}$ graphic representations significantly help monitoring, management, routine maintenance, study and promotion of a $\mathrm{CH}$ site and provides a way to transmit knowledge about heritage objects to future generations.

There is an increasing commitment to preserve and restore $\mathrm{CH}$, thereby fostering its better management, study or promotion. $\mathrm{CH}$ is a rich legacy for the current generation who have an undeniable responsibility to preserve it. Tangible Heritage becomes extremely important as a cultural, social and economic resource in modern societies. It is therefore necessary to continuously develop techniques in order to achieve a better understanding of its through-time evolution and improve maintenance approaches. Research, conservation and restoration of heritage assets are complex tasks that are being addressed from a multidisciplinary perspective: surveyors, architects, art historians, tourist promoters and advertising agents, amongst others. Since the footprint of time sometimes imposes terrible consequences on $\mathrm{CH}$, it often becomes necessary to not only recover the memory of original features of historical buildings, urban and landscape environments, but also understand its likely evolution. In this way, heritage legacy can be safeguarded for present and future generations, preventing future damage and aiding understanding of the current remains as an evolution of its original state.

Due to the advancement in technology, research and innovation improvements are increasingly noticeable, not only in the acquisition of data but also in the ability to include multiple complementary fields. Digital methods and techniques are able to link historical documentation data and disseminate them for a better understanding and perception of their evolution through time. With the aim of studying the current state and geometry of $\mathrm{CH}$ elements, numerous different geotechnologies can be used, from airborne to ground level, such as Airborne Laser Scanning (ALS), Mobile LiDAR Systems (MLS) and Terrestrial Laser Scanning (TLS), aerial and terrestrial photogrammetry, Global Navigation Satellite Systems (GNSS), etc. Historical datasets are extremely heterogeneous in terms of chronology, shape, style and structure, appearing as texts, paintings, engravings, old photographs, maps, etc. in analogue or digital formats. Therefore, it is necessary to establish schemes to order and clarify the current status regarding multifarious data acquisition and fusion for $\mathrm{CH}$ management at its different scales. It is also 
necessary to consider different data sources and their nature (e.g. metric or non-metric), as well as the final aim of 4D reconstruction and visualization.

\section{MULTI-SOURCE DATA FUSION}

Multi-source data fusion is one of the main challenges to face $4 \mathrm{D}$ reconstruction and visualization of $\mathrm{CH}$. This implies providing a solution for the combination of mixed data sources (both metric and non-metric) with the aim of creating timevarying representations. For this purpose, the suitability of the different sources of metric data should be systemized according to the $\mathrm{CH}$ object size and its complexity.

For a better understanding of the approach proposed by this paper to perform $\mathrm{CH} 4 \mathrm{D}$ analysis, it is helpful to group $\mathrm{CH}$ assets according to their characteristics. One of the most common classifications of $\mathrm{CH}$ studies (Kraak and Ormeling, 2011) is based on categorization according to the size of the element under study or scale range at the following levels: artefact, architectural, urban and rural landscapes (Table 1). This classification may seem unsophisticated, but the addition of other variables would complicate the classification and would result in confusion when describing the approach.

\begin{tabular}{ll}
\hline CH category & Scale ranges \\
\hline Artefact & From $1: 1$ to $1: 5$ \\
Architectural & From $1: 10$ to $1: 100$ \\
Urban landscape & From $1: 100$ to $1: 1000$ \\
Rural landscape & From $1: 100$ to $1: 5000$
\end{tabular}

Table 1. Typical scale ranges for each of the $\mathrm{CH}$ categories commonly established (adapted from Kraak and Ormeling, 2011).

Here, the different categories of $\mathrm{CH}$ assets are specified in order to assist inventory compilers and users in determining the appropriate procedures to be followed. Leaving aside "artefact" scale (although commonly considered in the field of $\mathrm{CH}$, it has not been included within the range of scales studied in this paper), three main categories of $\mathrm{CH}$ assets have been established: "rural landscape", "urban landscape" and "architectural" scales. The term "rural landscape" is applied to those cases where an extensive rural area exists. If the study case has a similar extension area to rural landscape cases, but instead of a rural environment it exists in urban space, the term "urban landscape" is adopted. Finally, "architectural" scale refers to those cases where the object in question focuses on a larger scale than in the two previous cases and where the $Z$ axis is predominant (buildings, monuments, sculpture statues, etc.).

\subsection{Overview of time-varying representations}

The 4D model generation that has to be implemented in $\mathrm{CH}$ can cover a large variety of situations, depending on the type of temporal analysis that is required (Figure 1).

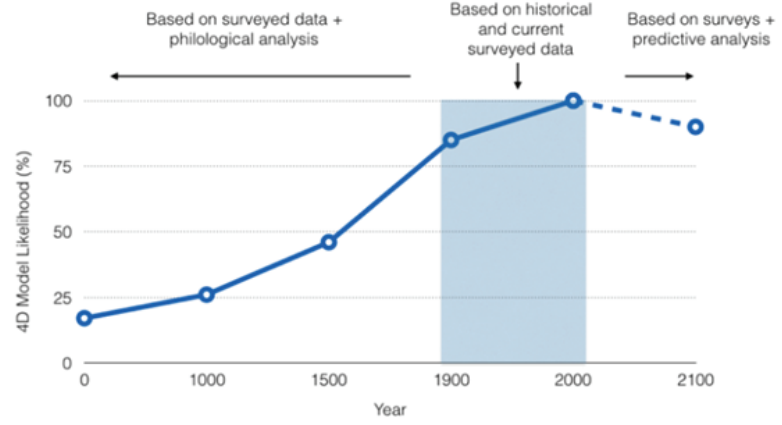

Figure 1. Three cases of time-dependent $3 \mathrm{D}$ analysis covered within the framework of the analysis of our legacy through time.

With reference to Figure 1, it is possible to identify three typical 4D model generations that can be produced for any $\mathrm{CH}$ asset:

a. Reconstruction of the diachronic evolution of a structure or environment that does not exist any longer, possibly with the exclusion of a few archaeological remains. This is generally associated to lost heritage, where partial traces arrived to the current age and will require the combination of surveyed data and philological analysis;

b. Reconstruction of the diachronic evolution of a structure or environment based on the historical analysis of data acquired on physically accessible assets at the current or a previous time (the blue highlighted area in Figure 1);

c. Prediction of the diachronic evolution of a structure or environment into the future based on the historical analysis of data acquired on physically accessible assets at different times.

Although all cases involve diachronic representation, the first and second cases ( $a$ and b) differ in the way in which 3D data can be collected, implying different concepts of 3D data integration:

1. In cases $b$ and $c$, the creation of the time-varying representations is based on rigorous metric $2 \mathrm{D}$ or $3 \mathrm{D}$ data, derived from a wide variety of sensors in physically accessible scenarios at different stages. For example, 3D models acquired at different times of a collapsing building surveyed during its deterioration;

2. In case a, the creation of time-varying documents or representations are based on the use of mixed sources, both metric and non-metric. For example, 3D reconstruction of a roman building at different stages according to both the 3D scanning of its remains and to historical sources.

In all cases, the concept of data fusion covers different aspects that are discussed below.

\subsection{Previous work on time-varying representations}

4D analysis based on real data captured on-site has been carried out mainly at urban levels due to the general availability of historical aerial images that allow the analysis of urban transformation and 4D modelling (Patias et al., 2011; Adami, 2015). The automatic processing of historical aerial images is not a trivial task, since it involves the recovery of unknown parameters (Redecker, 2008), which could yield geometric errors (Nocerino et al., 2012), and therefore mislead subsequent 4D analysis. For large $\mathrm{CH}$ sites, the optimal solution comes from the multi-source data sensor integration (Guidi et al., 2009), where the different methods and techniques balance their own drawbacks to reach an efficient solution (Gonizzi Barsanti 
et al., 2012). An example of the spatial-temporal analysis of a rural landscape is shown in Modica et al. (2011) where several geomatics technologies were employed, being one of the most efficient in the remote sensing imagery (Ratcliffe et al., 2004). For $\mathrm{CH}$ elements of reduced dimensions, such as buildings or archaeological remains, the reconstruction of temporal evolution is carried out on the basis of metric/non-metric data, such as historical drawings (Nocerino et al., 2014).

Besides reality-based models, in recent years reconstructive models have also assumed an interesting role, for the possibility to visualize architecture that no-longer-exists. This is achieved through an analytical process based on the integrated knowledge of historical sources and real 3D data. For more than two decades researchers have discussed the use of virtual reconstructions of environments that no-longer-exist as an instrument for the interactive interpretation of archaeological ruins or heavily stratified archaeological sites (Barcelo et al., 2000), for the presentation of generic cultural sites (Maver, 2001) and even for new archaeological discoveries (Frischer et al., 2008).

In order to define a shared standard based on strict methodologies, different relevant initiatives have been suggested by the Computer Applications and quantitative methods in Archaeology - Virtual Archaeology Special Interest Group (CAA-VASIG), the Cultural Virtual Reality Organization (CVRO) and from the European project EPOCH (www.epoch-net.org). Some of these contributions have been reported in the London Charter (www.londoncharter.org), the main aim of which is establishing internationally-recognized principles for the use of computer-based visualization by researchers, educators and Cultural Heritage organizations. On the basis of such criteria, several 4D projects have been developed that have integrated $3 \mathrm{D}$ data capture of a contemporary scene with 3D data of the same site reconstructed from paintings representing rigorous perspective views (ElHakim et al., 2008), or from plans giving the horizontal footprint of a building and drawings for reconstructing the elevation (Guidi et al. 2011). Moreover, other 3D data has been extracted from written sources describing different historical stages of a building (Micoli et al., 2013), or using the size of actual excavated decorations and the knowledge of specific rituals for adding geometrical constraints to the geometrical reconstruction of a religious building (Guidi et al., 2014).

\subsection{Overview of metric data sources}

The 3D digital reconstruction and documentation of existing $\mathrm{CH}$ assets for their next $4 \mathrm{D}$ visualization is a complex task that typically involves a hybrid approach to fuse heterogeneous datasets (Fai et al., 2011). The use of integrated approaches and technologies represents a crucial aspect since $\mathrm{CH}$ elements and sites usually present peculiarities that do not allow the definition of standard methodologies and procedures for every situation and context that it is possible to find. In this context, many interesting applications have been developed in recent years (Guidi et al., 2009, Callieri et al., 2011). Investigations have confirmed, on the one hand, that the workflow is often complex and requires expert assessment. On the other hand, it has been shown that the optimization of final models is the best practice. In order to overcome these critical aspects, the geotechnologies that can be used and combined in order to acquire 3D information of $\mathrm{CH}$ are classified according to the scale of the study (Figure 2).

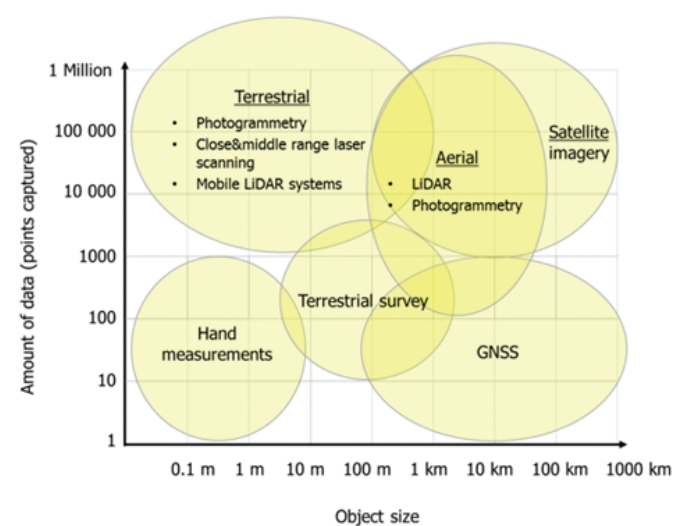

Figure 2. Three dimensional survey techniques characterised by scale and object size (derived from Böhler et al., 2001).

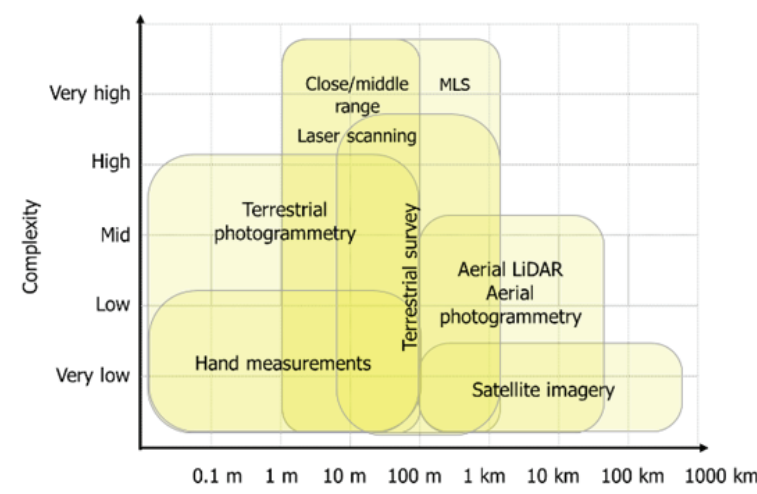

Object size

Figure 3. Three dimensional survey techniques characterised by complexity and object size (adapted from Böhler et al., 2001).

Due to their ability to collect large volumes of 3D data in a rapid and accurate way, range-based techniques such as TLS, MLS and ALS allow the acquisition of complex and versatile data that can be used in different contexts in a non-contact and non-destructive manner. On the other hand, image-based techniques such as terrestrial or aerial photogrammetry (onboard airborne systems such as gliders, trikes, drones, etc.) including automated implementations of Structure from Motion (SfM) algorithms, can provide valuable spatial object models adding high-quality photographic texture information. SfM approaches allow the reconstruction of $3 \mathrm{D}$ scenes (structure) and camera pose (motion) through sets of $2 \mathrm{D}$ images. The process mainly consists of image matching, camera orientation and self-calibration, and dense point cloud generation. These processes are based on a projective geometry and include the derivation of interior and exterior orientation parameters of the taking cameras. In computer vision, in contrast to the photogrammetric field, automation is more important than accuracy, however cooperation between both disciplines is allowing a compromise to be reached.

The continual fall in prices of both range and imaging sensors now offers the possibility for the digitalization of sites and objects to become more widespread, and is stimulating investigations into the possibilities of sensor and data integration with the aim of optimizing final results. In this context, one of the goals of analysing $\mathrm{CH}$ through time is to provide a methodology that can benefit from the advantages offered by both laser scanning and photogrammetry. The challenge is to define processes and methodologies to integrate different approaches and solutions within typical multiscale heritage contexts. 


\begin{tabular}{|c|c|c|c|}
\hline & Rural Landscape & Urban Landscape & Architectural \\
\hline Passive Systems & $\begin{array}{l}\text { - High-medium resolution satellite } \\
\text { imagery } \\
\text { - Aerial photogrammetry } \\
\text { - GNSS }\end{array}$ & $\begin{array}{l}\text { - Aerial photogrammetry } \\
\text { - Terrestrial photogrammetry } \\
\text { - GNSS }\end{array}$ & $\begin{array}{l}\text { - Close-range aerial } \\
\text { photogrammetry } \\
\text { - Terrestrial photogrammetry } \\
\text { - GNSS }\end{array}$ \\
\hline Active Systems & $\begin{array}{l}\text { - Airborne Laser Scanning } \\
\text { - Mobile LiDAR Systems }\end{array}$ & $\begin{array}{l}\text { - Airborne Laser Scanning } \\
\text { - Mobile LiDAR Systems } \\
\text { - Long-medium range TLS } \\
\text { - Total stations }\end{array}$ & $\begin{array}{l}\text { - Long-medium range TLS } \\
\text { - Total stations }\end{array}$ \\
\hline
\end{tabular}

\begin{tabular}{lll}
\hline \multicolumn{1}{c}{ Scanning system } & \multicolumn{1}{c}{ Use } & Typical accuracy and operating range \\
\hline ALS & To map and prospect landscapes (including forest areas) & $\begin{array}{l}\geq 0.05 \mathrm{~m}+(\mathrm{depending} \text { on the parameters } \\
\text { of the survey) } / 100 \mathrm{~m}-3500 \mathrm{~m}\end{array}$ \\
Time of flight TLS & $\begin{array}{l}\text { To survey building façades and interiors, resulting in line drawings } \\
\text { (with support data) and surface models } \\
\text { To survey building façades and interiors, resulting in line drawings }\end{array}$ & $\begin{array}{l}\text { - } 6 \mathrm{~mm} \text { at ranges up to several hundred } \\
\text { meters }\end{array}$ \\
Phase-shift TLS & $\begin{array}{l}\text { (with support data) and surface models - particularly where rapid } \\
\text { data acquisition and high point density are required }\end{array}$ & $5 \mathrm{~mm}$ at ranges up to $50-100 \mathrm{~m}$ \\
MLS & $\begin{array}{l}\text { For city models, as-built documentation, monitoring environmental } \\
\text { changes, etc. }\end{array}$ & $10-50 \mathrm{~mm} / 100-200 \mathrm{~m}$ \\
\hline
\end{tabular}

Table 3. Laser scanning techniques used in CH management activities (adapted from Barber et al., 2006).

\subsection{Fusion of metric and non-metric data}

In addition to 3D digital surveys, performed through an integration of technologies as described previously, the reconstruction of structures that are nowadays partially or totally destroyed might be based on a multiplicity of historical documents that very often have non-metric properties, such as:

- historical maps;

- ancient photographs;

- ancient drawings and sketches;

- ancient paintings, which represent tilted views of nonexisting scenarios (especially useful for the height reconstruction of buildings);

- other historical iconography;

- written descriptions on ancient texts;

- indirect geometrical deductions.

The first step for historical reconstruction starts with redesigning in CAD software an updated version of the documents (e.g. ancient maps), or applying some perspective estimation deduced from the old graphical representation. Such historic data lack in objectivity and mutual coherence due to reasons such as: the line thickness of old drawings; the presence of errors in ancient surveys and restitutions; the distortions introduced by the different reproduction devices used to generate the current paper instance of a particular document; improper conservation of the original document (e.g. due to humidity/temperature stress), that may give a low accuracy in the final representation. Such lack in objectivity requires a further step for adaptation from rough graphic output of the traditional representation technique to the precise CAD drawing, maintaining a proportional coherence between single pairs and the whole (Guidi and Russo, 2011). The presence of non-coherent variation in dimension and proportion among drawings of different historical periods can be compensated by non-uniform scaling. The correction of these traditional representations is derived from real elements in the structures that can still be precisely measured (e.g. a building footprint).

In addition, indirect estimations may give additional geometrical information as to the volumes of some structures. For example, the amount of debris collected during an archaeological excavation can be directly measured if the excavation is still in progress, or may be documented by old images from an old excavation. Similarly, ancient cadastral data may give the expected population in a certain area of a city, providing a rough estimate as to the number of inhabitants to be accommodated, and by inference the number of floors for certain buildings. Finally, information about rituals may ensure the complete occlusion of a holy building with respect to a determined structure, providing indirect additional constraints to be mixed with the other clues for estimating the possible height of no-longer-existing buildings (Micoli et al., 2013).

\section{CURRENT GEOTECHNOLOGIES FOR DATA ACQUISITION}

The most important parameters related to the scale of the $\mathrm{CH}$ assets and the suitability of different geotechnologies for data acquisition are described in depth in this section. Nowadays, two main data acquisition methods are used to generate 3D models of the current state of $\mathrm{CH}$ : image-based and range-based techniques, which use passive and active sensors respectively. Depending on the particular case study and the working scale different geomatic systems are recommended, as Table 2 shows. Some of the geotechnologies classified as passive or active systems in Table 2 can be defined as hybrid systems, for example MLS which integrates navigation (GNSS/INS), range (laser scanners) and imaging (cameras) sensors, but they are kept in the active-passive dichotomy for the ease of understanding. 
Table 3 specifies the suitability of each of the laser scanning techniques concerning their applicability to $\mathrm{CH}$ studies and within the framework established by the analysis of $\mathrm{CH}$ through time. Note that short-range or triangulation-based laser scanners have not been considered since they are commonly used to document small objects, at the artefact scale, with a higher level of detail. Aside from the working scale, there are certain parameters that will influence data acquisition planning and data processing and, therefore, will provide the best suitability towards using a specific technology. Table 4 shows the most important parameters. The network design (distances, number of station points, angles, etc.) will depend on these parameters. A qualitative classification has been applied for the three different scale levels. In this way, each $\mathrm{CH}$ scale is scored from 1 to 3, meaning "low", "medium" and "high", respectively, depending on the level required for each of the variables (Table 4).

\begin{tabular}{llll}
\hline Parameters & Rural l. & Urban l. & Archit. \\
\hline Extension of the area to be measured & $\bullet \bullet \bullet$ & $\bullet \bullet$ & $\bullet$ \\
Complexity of the CH element & $\bullet$ & $\bullet \bullet$ & $\bullet \bullet \bullet$ \\
Amount of data captured & $\bullet \bullet$ & $\bullet \bullet \bullet$ \\
Time consumed in data acquisition & $\bullet$ & $\bullet \bullet$ & $\bullet \bullet \bullet$ \\
$\quad \begin{array}{l}\text { and processing } \\
\text { Complexity in data integration }\end{array}$ & $\bullet$ & $\bullet \bullet \bullet$ & $\bullet$ \\
Level of detail & $\bullet$ & $\bullet \bullet$ & $\bullet \bullet \bullet$ \\
Precision required & $\bullet$ & $\bullet \bullet$ & $\bullet \bullet \bullet$ \\
Spatial resolution & $\bullet$ & $\bullet \bullet$ & $\bullet \bullet \bullet$ \\
Scoring meaning: & $\bullet$ High & $\bullet$ & Medium
\end{tabular}

Table 4. Scoring of parameters that influence acquisition, processing and integration tasks depending on the $\mathrm{CH}$ scale of study.

Table 4 highlights that, broadly speaking, architectural studies are the most restrictive in terms of the evaluated features, since the process of documentation in such an environment is more complex. Intensive processing and modelling steps are necessary given the high spatial resolution of data captured, which is explained by the level of detail required. For that reason, it sums to the highest total score of the three levels of scale considered. In addition, the most remarkable factor for rural landscape studies is the extension of the area to be documented since, generally, this is the largest of the three scales established. Finally, regarding urban environments, it is worth noting that it essentially represents an intermediate case between rural landscape and architectural scales. For urban landscape scale studies, a greater amount of data can be collected due to its inherent man-made characteristics. Therefore, a greater variability of suitable sensors has to be used, as shown in Table 2. The raw data that is acquired has to be transformed into more usable products through multiple processing steps.

\section{4D DATA MANAGEMENT}

Once a 4D dataset has been collected and registered, it is necessary to use specific tools and methods that will allow their integration in order to undertake $\mathrm{CH}$ management, analysis and visualization tasks. Although Building Information Modelling (BIM) is used in modern architecture for representing buildings, not only graphically but also as a hierarchy of functional parts, its concepts have been ported to the world of ancient buildings, leading to the term Heritage BIM (HBIM) (Murphy et al., 2009). This also requires an interpretation of the collected $3 \mathrm{D}$ data that have to be subdivided in a semantically logical way (De Luca et al., 2006).

GIS and BIM models have the ability to integrate digital data and representations of the real world from different systems. They are not mutually exclusive but rather complementary methods that are used to support decision-making and problem solving depending on the $\mathrm{CH}$ scale. It is not easy to reach a global consensus in this matter; however, between rural landscape and architectural scales the difference becomes more noticeable. For these last two scales, a GIS and a BIM model, respectively, are the most suitable solutions to integrate the processed data. Meanwhile, for an urban landscape scale there is less of a consensus to decide which of the two systems is the most appropriate in this regard. This is because BIM is used in a relatively micro level of the real world (architecture and handles mainly indoor data) linked to three dimensional solids and surfaces; and GIS is used in macro level of the real world (terrain, land parcels and outdoor data), traditionally paired with two dimensional data.

As Table 5 shows, BIM is commonly used to navigate within a building and its hierarchy structure for planning, design, construction and management. 3D modelling is just one aspect of BIM that has hogged the limelight, but its real strength and power lies in the knowledge database. This is the reason for its use in conjunction with other software tools to deliver quick and reliable information in areas of sustainability, structural analysis and reconstruction (Sah and Cory, 2008).

Meanwhile, the strength of GIS lies in the ability to provide data referenced by spatial or geographic coordinates. Thus, a GIS is both a database system with specific capabilities for spatially referenced data, as well as a set of operations to spatial data management. High-level geospatial datasets are required together with a relational database. The optimal situation would be to encompass the advantages of both systems, so that a final toolset would allow an easy data translation that optimizes the capabilities of the separate software and methods. In this regard, Irizarry et al. (2013) integrated BIM and GIS into a unique system, which enabled tracking of the supply chain status and providing warning signals to ensure the delivery of materials. GIS is widely used for integrating, visualizing and analysing information about real world assets, such as buildings and transportation infrastructure, with surrounding context that may include environmental, demographic, structural, and scientific information (de Laat and Van Berlo, 2011).

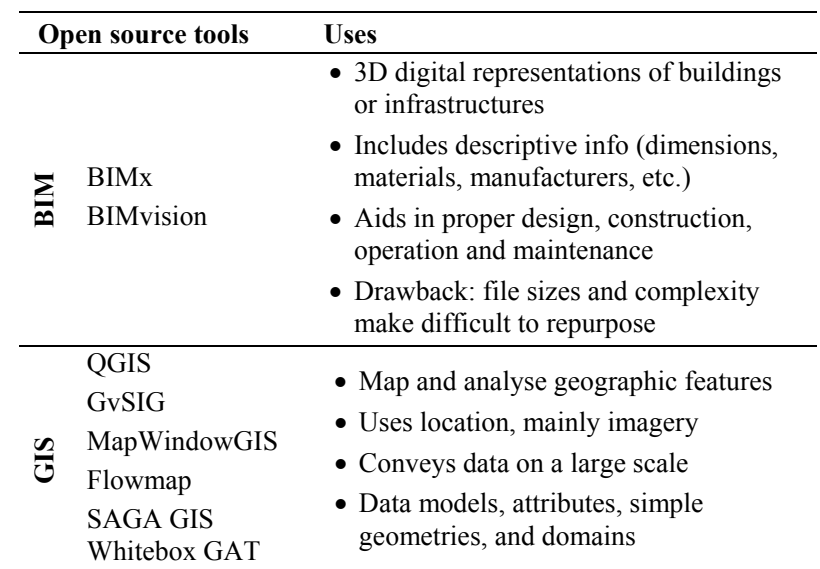

Table 5. Most common uses of BIM and GIS, and some of the open source software available. 
In the GIS world, the term GeoDesign has been used to refer to a scenario-based planning process that applies decision making to spatial problems in the context of local and regional features and characteristics. BIM derived originally from CAD more than from GIS. Initially CAD and GIS were technologically very close. However, their development went in different directions and now they are very far from each other, not only in the scale and Level of Detail (LoD), but also differences in semantic, terminology, techniques, coordinates, etc. (Hijazi et al., 2010). A major difference is the lack of primitives in GIS modelling (used in CAD) and, on the other hand, usually a lack of topology in CAD which is basic in GIS. Therefore, the problem of integration between BIM, as an extension of CAD, and GIS is essential in the use of $4 \mathrm{D}$ modelling in $\mathrm{CH}$. An interesting approach for the integration can be found in Hijazi et al., (2010) where the BIM4GEOA concept was presented. BIM and GIS are databases, however different, and the integration platform can be built using the database platform; for example spatial open source Postgres/PostGIS (Hijazi et al., 2010, Boysen et al., 2014). In the literature on the integration of BIM and GIS, authors commonly use standard Industry Foundation Classes (IFC), as an input data format for BIM and Open Geospatial Consortium (OGC, Web Map Service - WMS, Web Feature Service - WFS, Geography Markup Language GML etc.) as a standard input GIS format. BIM/GIS integrated in one spatial database could be easily published on a web platform to enable visualization (Section 5) and browsing by different end users.

\section{VISUALIZATION}

Nowadays, hardware and software resources are used to create new applications, platforms and systems with the aim of visualizing and managing large digital datasets in real-time. In some cases, they are not limited to simple visualizations with animation sequences of 3D models but rather they can be linked to databases in order to arrange the digital information for different purposes, such as studies, analyses (queries), preservation, simulations and divulgation. In addition, they can take advantage of well-known 3D visualization technologies such as Virtual Reality (VR) and Augmented Reality (AR).

\subsection{Devices}

Although virtual technologies and related devices such as Head Mounted Displays (HMD) have been well known for two decades in military and medical simulations (Billinghurst, 1998), recent low-cost developments in the gaming industry have strongly revamped these technologies, proposing low cost devices for VR and AR with functionalities very similar to the original ones at a fraction of the original cost. For this reason they are bringing VR to consumers worldwide, and not only in the video gaming area. They are becoming increasingly popular thanks to their potential for natural and intuitive user interaction. Among existing systems for $\mathrm{CH}$ virtual applications, it should be noted that VR is very useful to represent $\mathrm{CH}$ through time. VR is a computer-simulated life or immersive multimedia experience where the real world is replaced by a virtual one and the physical presence is simulated. VR begins to play an important role in museums where different historical scenarios are simulated, giving the possibility to explore them again (Loizides et al., 2014). VR is paving the road to a new and exciting way to communicate historical information, where the user is completely immersed in an interactive world.
Game engine software such as Unity (commercial) or Blender VR and Demotride viewer (both freeware) allows the integration and management of external devices with the aim to integrate the user's body movements during the visual simulation. VR devices can also be integrated among them to interact and navigate in a complex 4D (3D + time) scene, as well as to provide access to digital media contents of $\mathrm{CH}$ monuments and sites. In this way, fragile $\mathrm{CH}$ environments such as rural and urban landscapes as well as architectural places can be virtually visited, studied and analysed without being damaged. Many applications have been already produced in the $\mathrm{CH}$ field for simulating archaeological sites in a shared virtual environment (Vote et al., 2002), for virtually accessing an underwater archaeological site (Haydar et al., 2011), or allowing different experts and scholars to interact with small $\mathrm{CH}$ elements or sub-elements in AR (Fernández-Palacios, et al., 2015).

\subsection{Data optimization}

Despite recent technological breakthroughs, limitations still exist regarding the VR visualization and management of huge datasets with a fluent real-time interaction. In fact, 3D recording techniques produce optimal and accurate photo-realistic 3D models which generally have very large data sizes. The management of both digitalized historical collected data and current datasets is therefore not a trivial task, due to their size and the high level of processing requirements. Therefore, an optimization approach for the correct 4D VR visualization and management in real-time is needed, covering the following tasks:

- Optimization of digitalized analogue format data and 2D digital data:

- Metadata optimization;

○ Digitalization: simplify resolution;

- Database optimization.

- Optimization of 3D datasets:

○ 3D models segmentation;

- Geometric optimization: normal and displacement maps (for the high and low resolution details respectively);

- Radiometric optimization: texture simplification, unwrap techniques.

The need to optimize data is mainly due to the employment of polygonal 3D models. These are often composed of millions of polygons and high resolution images. Due to their large size, they are difficult to handle on standard devices, firstly because of the time required to upload and process data associated with large polygonal 3D models and large 3D datasets. Secondly, the real-time visualization of $3 \mathrm{D}$ models is constrained by a device's graphics card performance. It is therefore necessary to optimize the 3D models. From a geometric point of view, the number of vertices will be reduced to optimize the geometry. However, it has been demonstrated that appropriate 3D processing may give up to 25 times less polygons of the original acquired 3D model, still maintaining the same geometrical and visual aspect (Guidi et al., 2016). Normal and displacement maps will therefore be used to maintain all the relevant details and textures. From a radiometric point of view, UV unwrapping techniques will enable the optimization of texture files.

In order to carry out the simplification and optimization tasks to visualize the different $3 \mathrm{D}$ products through the Web, it is possible employ open source libraries, such as Cesium (WegGL, 2016), or Geoweb 3D (GeoWeb, 2016). Moreover, the data exchange and rendering it is an issue under study, since 
it could improve the data transmission and data streaming by means of a Level of Detail (LoD) visualization (Limper et al., 2014). An implementation of the above-mentioned simplification, optimization for web visualization of a large extension archaeological site (landscape scale) is shown in Torres-Martínez, et al., (2016).

Once the datasets are optimized, in order to recreate a 4D VR simulation, the 3D models of interest (current and past or future hypothetical situation) must be referenced to a unique coordinate system (local or global), scaled and overlapped with the same origin and orientation and used to create temporal VR visualizations to highlight changes. In this way, VR technologies and devices allow users to navigate and visualize the entire $\mathrm{CH}$ site, through time, which comprehensively reveals the detailed diachronic evolution (e.g. restorations, construction phases, demolitions, influence of natural phenomena, etc.) of the elements under study. The user can emulate and visualize the sequence of construction operations involved as a coherent and unified process. Virtual technologies (Virtual, Augmented and Mixed Reality) and devices (headset, 3D sensor, etc.) depend on the $\mathrm{CH}$ scale study case. In any case, for urban and/or rural landscapes as well as architectural cases studies, VR applications present an unparalleled solution to visualize and manage these kind of data.

\section{CONCLUSIONS}

Representing the relationship between time and space provides a powerful mechanism to visualize and communicate design intent. It can be useful not only to study and analyse our past, but also to foresee possible risks in the future. This manuscript provides a comprehensive overview to conducting studies of $\mathrm{CH}$ assets over time for three working scales and different environments: rural landscape, urban landscape and architectural scales. It serves as an initial guide for organizing all the tasks, from collecting historical documentation, acquiring current data of the $\mathrm{CH}$ element, processing to visualizing results. It is oriented to perform studies through time, including the monitoring of $\mathrm{CH}$ assets that still exist or that no longer exist but whose time evolution left current remains on the landscape. Furthermore, an estimation of the time spent in each method phase is provided. Meanwhile, due to the wider scope of the topic addressed, this document is open to be supplemented, especially in those method stages containing greater ambiguity and variability for the requirements of each specific case study and data to be collected. Therefore, the analysis of $\mathrm{CH}$ through time, as described here, is open to be enhanced with other specific studies depending on the particular $\mathrm{CH}$ scale.

\section{ACKNOWLEDGEMENTS}

The authors wish to acknowledge the support of the European Union Joint Programming Initiative on Cultural Heritage (JPI$\mathrm{CH}$ ), for funding the project Cultural Heritage Through Time (CHT2), through national research authorities: Spanish Ministry of Economy and Competitiveness, UK Arts and Humanities Research Council (AHRC, award number AH/N504440/1) and Polish Ministry of Culture and National Heritage.

\section{REFERENCES}

Adami, A., 2015. 4D city transformations by time series of aerial images. In: The International Archives of the
Photogrammetry, Remote Sensing and Spatial Information Sciences, Avila, Spain, Vol. XL-5/W4, pp. 339-344.

Barber, D. M., Dallas, R. W., Mills, J. P., 2006. Laser scanning for architectural conservation. Journal of Architectural Conservation, 12(1), pp. 35-52.

Barcelò, J.A., Forte, M., Sanders D.H., 2000. Virtual Reality in Archaeology. BAR International Series 843, Archeopress, Oxford.

Billinghurst, M., Bowskill, J., Dyer, N., Morphett, J., 1998. An evaluation of wearable information spaces. In: VRAIS '98: Proceedings of the Virtual Reality Annual International Symposium, p. 20. IEEE Computer Society, Washington, DC, USA.

Boehler, W., Hein, G., Marbs, A., 2001. The potential of noncontact close range laser scanners for cultural heritage recording. In: XVIII CIPA Symposium, Postdam, Germany

Boysen, M., de Haas, C., Lu, H., Xie, X., Pilvinyte A., 2014. Constructing indoor navigation systems from digital building information. In: IEEE 30th International Conference on Data Engineering, pp. 1194-1197.

Callieri, M., Chica, A., Dellepiane, M., Besora, I., Corsini, M., Moyés, J., Ranzuglia, G., Scopigno, R., Brunet, P., 2011. Multiscale acquisition and presentation of very large artifacts: The case of portalada. Journal on Computing and Cultural Heritage, 3(4), 14:1-14:20.

de Laat, R., Van Berlo, L., 2011. Integration of BIM and GIS: The development of the CityGML GeoBIM extension. In: Advances in 3D geo-information sciences, pp. 211-225.

De Luca L., Veron P., Florenzano M., 2006. Reverse engineering of architectural buildings based on a hybrid modeling approach, Computers \& Graphics, 30, pp. 160-176.

El-Hakim, S., Lapointe, J.-F., Whiting, E., 2008. Digital reconstruction and 4D presentation through time. In: SIGGRAPH 2008, Los Angeles, California.

Fai, S., Graham, K., Duckworth, T., Wood, N., Attar, R., 2011. Building information modelling and heritage documentation. In XXIII CIPA Symposium, Prague, Czech Republic, pp. 12-16.

Frischer, B., Dakouri-Hild, A., 2008. Beyond Illustration. 2D and 3D Digital Technologies as Tools for Discovery in Archaeology, BAR International Series 1805, Oxford, 168 pp.

Geoweb3d-3D GIS Visualization. Available online: http://www.geoweb3d.com/ (accessed on 15 September 2016).

Gonizzi Barsanti, S., Remondino, F., Visintini, D., 2012. Photogrammetry and Laser Scanning for archaeological site 3D modeling - Some critical issues. In: 2nd Workshop on 'The New Technologies for Aquileia'. Aquileia, Italy.

Guidi, G., Frischer B., Lucenti I., 2007. Rome Reborn Virtualizing the Ancient Imperial Rome. In: The International Archives of the Photogrammetry, Remote Sensing and Spatial Information Sciences, Zurich, Switzerland, Vol. XXXVI-5/W4, pp. 1-4.

Guidi, G., Remondino, F., Russo, M., Menna, F., Rizzi, A., Ercoli, S., 2009. A multi-resolution methodology for the 3D modeling of large and complex archaeological areas. 
International Journal of Architectural Computing, 7(1), pp. 3955 .

Guidi, G., Russo, M., 2011. Diachronic 3D reconstruction for lost Cultural Heritage. In: The International Archives of the Photogrammetry, Remote Sensing and Spatial Information Sciences, Trento, Italy, Vol. XXXVIII-5/W16, pp. 371-376.

Guidi, G., Russo M., Angheleddu D., 2014. 3D Survey and Virtual Reconstruction of Archeological Sites. Digital Applications in Archaeology and Cultural Heritage, 1(2), 5569.

Guidi, G., Angheleddu, D., 2016. Displacement Mapping as a Metric Tool for Optimizing Mesh Models Originated by 3D Digitization. Journal on Computing and Cultural Heritage, 9(2), pp. 9:1-9:23

Hijazi, I., Ehlers, M., Zlatanova, S., 2010. BIM for geo-analysis (BIM4GEOA): set up of 3D information system with open source software and open specification (OS). In: The International Archives of the Photogrammetry, Remote Sensing and Spatial Information Sciences, Berlin, Germany, Vol. XXXVIII-4/W15, pp. 45-49.

Haydar M., Roussel D., Otmane S., Mallem M., 2011. Virtual and augmented reality for cultural computing and heritage: a case study of virtual exploration of underwater archaeological sites. Virtual Reality, 15 (4), pp. 311-327.

Irizarry, J., Karan, E. P., Jalaei, F., 2013. Integrating BIM and GIS to improve the visual monitoring of construction supply chain management. Automation in Construction, 31, pp. 241254.

Jiménez Fernández-Palacios, B., Nex, F., Rizzi, A., Remondino, F., 2015. ARCube - The Augmented Reality Cube for Archaeology. Archaeometry, 57(S1), pp. 250-262.

Kraak, M. J., Ormeling, F., 2011. Cartography: visualization of spatial data. Guilford Press. USA.

Limper, M.; Thöner, M.; Behr, J.; Fellner, D.W., 2014. SRC-a streamable format for generalized web-based $3 \mathrm{D}$ data transmission. In: 19th International ACM Conference on 3DWeb Technologies, Vancouver, BC, Canada, pp. 35-43.

Loizides, F., El Kater, A., Terlikas, C., Lanitis, A., Michael, D., 2014. Presenting Cypriot Cultural Heritage in Virtual Reality: A User Evaluation. Lecture Notes in Computer Science, 8740, pp. $572-579$.

Maver, T. 2001. Virtual Heritage: Reconstructing the Past, Reconfiguring the Future. In: Seventh International Conference on Virtual Systems and Multimedia, Berkely, California, pp. 168-176.

Micoli, L., Guidi, G., Angheleddu, D., Russo M., 2013. A multidisciplinary approach to 3D survey and reconstruction of historical buildings, In: Digital Heritage International Congress (DigitalHeritage), Marseille, France, pp. 241-248.

Modica, G., Vizzari, M., Pollino, M., Fichera, C. R., Zoccali, P., Di Fazio, S., 2012. Spatio-temporal analysis of the urban-rural gradient structure: an application in a Mediterranean mountainous landscape (Serra San Bruno, Italy). Earth System Dynamics, 3(2), pp. 263-279.
Murphy M, McGovern E, Pavia S., 2009. Historic Building Information Modelling (HBIM), Structural Survey, 27(4), pp. 311-327.

Nocerino, E., Menna, F., Remondino, F., 2012. Multi-temporal analysis of landscapes and urban areas. In: The International Archives of the Photogrammetry, Remote Sensing and Spatial Information Sciences, 2, Melbourne, Australia, Vol. XXXIXB4, pp. 85-90.

Nocerino, E., Fiorillo, F., Minto, S., Menna, F.,Remondino, F., 2014. A Non-Conventional Procedure for the 3D Modeling of WWI Forts. In: The International Archives of the Photogrammetry, Remote Sensing and Spatial Information Sciences, Riva del Garda, Italy, Vol. XL-5, pp. 457-464.

Patias, P., Kaimaris, D., Stylianidis, E., 2011. Change detection in historical city centers using multi-source data: the case of historical center of Nicosia - Cyprus. In: XXIII CIPA Symposium, Prague, Czech Republic.

Ratcliffe, I.C., Henebry, G.M., 2004. Using declassified intelligence satellite photographs with Quickbird imagery to study urban land cover dynamics: a case study from Kazakhstan. In: ASPRS Annual Conference, Denver, USA, pp. 198:1-198:10

Redecker, A.P., 2008. Historical aerial photographs and digital photogrammetry for impact analyses on derelict land sites in human settlement areas. In: The International Archives of the Photogrammetry, Remote Sensing and Spatial Information Sciences, Beijing, China, Vol. XXXVII-B8, pp. 510 .

Sah, V., Cory, C., 2008. Building information modeling: An academic perspective. In: IAJC-IJME 2008 International Conference. Nashville, Tennessee.

Torres-Martínez, J.A.; Seddaiu, M.; Rodríguez-Gonzálvez, P.; Hernández-López, D.; González-Aguilera, D., 2016. A MultiData Source and Multi-Sensor Approach for the 3D Reconstruction and Web Visualization of a Complex Archaelogical Site: The Case Study of "Tolmo De Minateda". Remote Sensing, 8(7), 550:1-550:25.

Vote, E, Feliz D A, Laidlaw D H, Joukowsky M S., 2002. Discovering Petra: Archaeological Analysis in VR. IEEE Computer Graphics and Applications, 22(5), pp. 38-50.

WebGL Virtual Globe and Map Engine. Available online: http://cesiumjs.org/ (accessed on 15 September 2016). 